\title{
First observations of GRS 1915+105 with e-VLBI
}

\author{
A. Rushton, ${ }^{* 1}$ R. E. Spencer, ${ }^{1}$ M. Strong, ${ }^{1}$ R. M. Campbell, ${ }^{2}$ S. Casey, ${ }^{1}$ R. P. Fender, ${ }^{3,4}$ \\ M. A. Garrett, ${ }^{2}$ J. C. A. Miller-Jones, ${ }^{4}$ G. G. Pooley, ${ }^{5}$ C. Reynolds, ${ }^{2}$ A. Szomoru, ${ }^{2}$ V. \\ Tudose $^{4,6}$ and Z. Paragi ${ }^{2}$ \\ ${ }^{1}$ The University of Manchester, Jodrell Bank Observatory, Cheshire SK11 9DA. \\ ${ }^{2}$ Joint Institute for VLBI in Europe, Postbus 2, 7990 A A Dwingeloo, The Netherlands. \\ ${ }^{3}$ School of Physics and Astronomy, University of Southampton, Highfield, SO17 1BJ \\ Southampton, UK. \\ 4 “Anton Pannekoek” Astronomical Institute, University of Amsterdam, Kruislaan 403, 1098 SJ \\ Amsterdam, The Netherlands. \\ ${ }^{5}$ University of Cambridge, Mullar Radio Astronomy Observatory, J. J. Thomson Avenue, CB3 \\ OHE Cambridge, UK. \\ ${ }^{6}$ Astronomical Institute of the Romanian Academy, Cutitul de Argint 5 RO-040557 Bucharest, \\ Romania
}

\begin{abstract}
We present results from the first successful open call e-VLBI science run, observing the X-ray binary GRS 1915+105. e-VLBI science allows the rapid production of VLBI radio maps, within hours of an observation rather than weeks. A total of 6 telescopes observing at $5 \mathrm{GHz}$ across the European VLBI Network (EVN) were correlated in real time at the Joint Institute for VLBI in Europe (JIVE). Throughout this, GRS 1915+105 was observed for a total of 5.5 hours, producing $2.8 \mathrm{~GB}$ of visibilities of correlated data. The peak brightness was $10.2 \mathrm{mJy}$ per beam, with a total integrated radio flux of $11.1 \mathrm{mJy}$.
\end{abstract}

VI Microquasar Workshop: Microquasars and Beyond

September 18-22 2006

Società del Casino, Como, Italy

\footnotetext{
*arushton@jb.man.ac.uk
} 


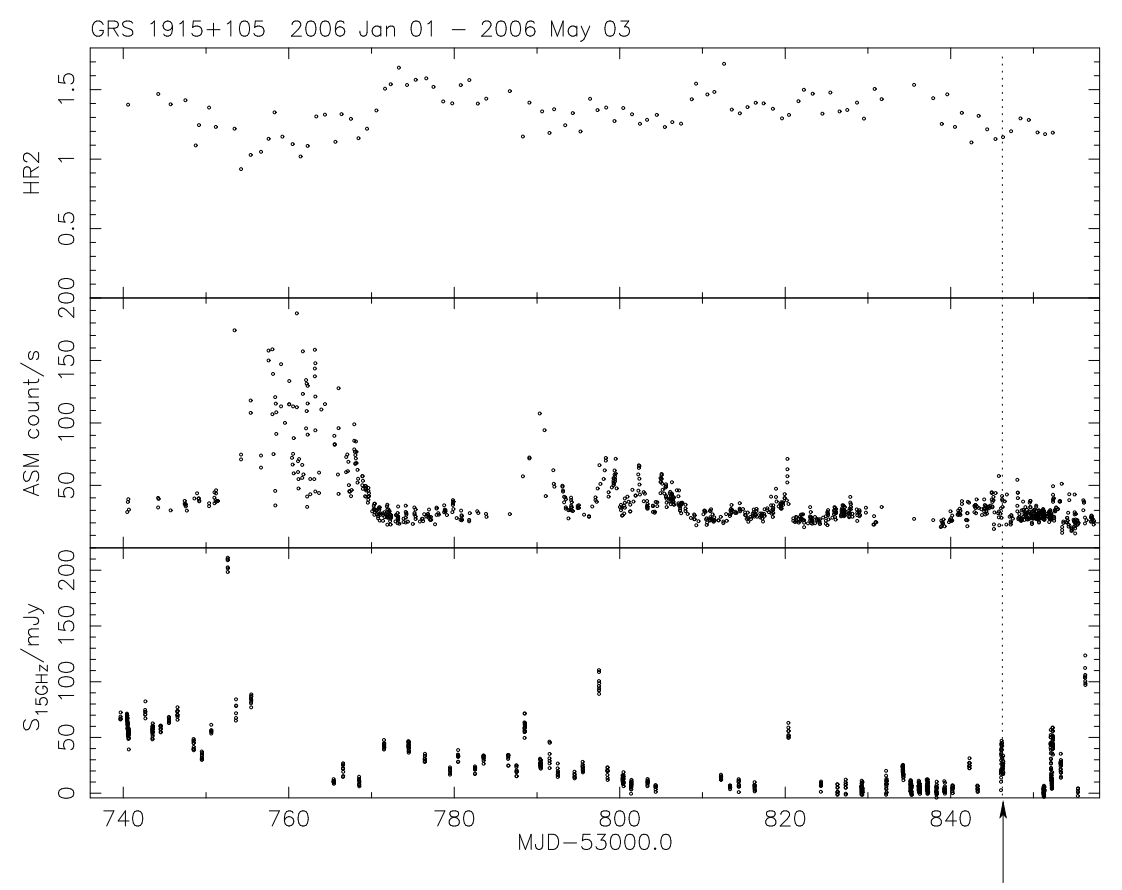

Figure 1: RXTE X-ray and Ryle telescope $15 \mathrm{GHz}$ flux density monitoring of GRS 1915+105, observed between 2006 Jan 01 and May 03. The data of the e-VLBI observation (MJD 53846), is marked by the dotted line. The top shows the X-ray spectral hardness ratio $\left(\frac{5-12 \mathrm{keV}}{3-5 \mathrm{keV}}\right)$, the middle shows the RXTE ASM count-rate and the bottom shows the $15 \mathrm{GHz}$ radio flux density.

\section{Introduction}

The use of the Internet for electronic very-long-baseline interferometry (e-VLBI) data transfer offers a number of advantages over conventional recorded VLBI, including improved reliability due to real time operation and the possibility of a rapid response to new and transient phenomena. Decisions on follow-up observations can be made immediately after the observation rather than delayed by potentially weeks due to problems in shipment of tapes/discs to the correlator. A number of recent test runs have shown that $128 \mathrm{Mbps}$ data rates can be obtained reliably to the 6 European telescopes; Cambridge, Jodrell Mk2, Medicina, Onsala, Torun and Westerbork, currently connected via national and international research networks to the EVN correlator at Joint Institute for VLBI in Europe (JIVE). Steps are currently being taken to improve the reliability of 256 and $512 \mathrm{Mbps}$ connections, and also develop $1 \mathrm{Gbps}$ transmission as part of the EXPReS ${ }^{1}$ project.

Microquasars are ideally suited for study by e-VLBI since they often have fhres associated with the ejection of radio emitting clouds in the form of jets. Time-scales of this emission are in the range of hours to days at $\mathrm{cm}$ wavelengths, and decisions about subsequent observations, need to be taken quickly.

The X-ray binary GRS 1915+105 was first discovered in 1992 [2] by the WATCH instrument on the GRANAT satellite. The system comprises a low mass, K-M III star [9] companion and a $14 \pm 4 \mathrm{M}_{\odot}$ black hole [8]. It was the first Galactic source observed to display superluminal

\footnotetext{
${ }^{1}$ see - http://www.expres-eu.org
} 
motion, and is well known for its rapid variability and strong variable radio flux. It spends the majority of its time in relative radio-quiescence, with low radio and X-ray brightness, and with a characteristic low/hard state X-ray spectrum. In such a state the source is thought to be 'jetdominated' [5], with a $\sim 50$ AU scale inner radio jet [3] present. Transitions to the soft state are often accompanied by strong radio flares with the ejection of a high velocity component out to distances of several hundred milliarcsecond or $\sim 10^{4} \mathrm{AU}$; these transitions have been studied by the VLA and MERLIN [12, 6, 11]. Long-term high sensitivity VLBI monitoring of motions in the core is necessary to understand how the inner jets relate to the larger scale ejections. This is not possible without the strategy in place enabling rapid decisions on follow-up VLBI observations.

Over the first few months in 2006, GRS 1915+105 has been consistently fhring in radio (Figure 1). A $300 \mathrm{mJy}$ (at $4.8 \mathrm{GHz}$ ) steep spectrum, optically thin flare was detected by the RATAN 600 telescope on $2006 \mathrm{Feb} 23$, suggesting that the source may have undergone a transition to the high / soft state. This triggered a MERLIN target of opportnity (ToO) on transient sources which detected another outburst in March 2006 (Miller-Jones et al. in prep.). One aim of the project was also to develop a strategy for rapid response (ToO) e-VLBI observations for when this technique is more mature.

\section{Observations and results}

On 2006 April 20 - 21 the e-EVN observed GRS 1915+105 at $4.994 \mathrm{GHz}$. The data were transferred from the telescope to the correlator using Mark 5A disk-based VLBI data systems. These units have been fitted with 1 Gbps Network Interface Cards which allow the units to transfer the telescope data to the correlator over the Internet and private optical networks at rates exceeding 100 Mbps. Production Internet connections for institutions within each participating country are provided and controlled by the local and national network providers. Most of the telescopes connect to the national networks, and then are connected to the GÉANT 2 network ${ }^{2}$ allowing panEuropean multi-gigabit connectivity. Further details on the Internet connections will be presented by Szomoru et al. (in prep.) and Strong et al. (in prep).

Each station sustained a transfer rate of 128 Mbps across the e-VLBI network. This transmission rate supports two $8 \mathrm{MHz}$ dual-polarisation basebands channels, providing a total bandwidth of $32 \mathrm{MHz}$. The observations were made using the phase-reference mode with a cycle of $5 \mathrm{~min}-$ utes on source and 3 minutes on the phase reference, J1925+1227. A bright compact radio source, J2002+4725 was used as a fringe finder, was scheduled at the beginning and toward the end of the observing run.

The radio image of GRS $1915+105$ on 2006 April $20-21$ is shown in Fig. 2 using a $u v$ weighting robustness parameter of 0 [1]. The source had a position of R.A. $19^{\mathrm{h}} 15^{\mathrm{m}}(11.548 \pm 0.001)^{\mathrm{s}}$ and Dec. $10^{\circ} 56^{\prime}(44.71 \pm 0.01)^{\prime \prime}(\mathrm{J} 2000)$. The position is consistent with that expected from the known proper motion [11]. The sources appears marginally resolved and was deconvolved from the beam using the AIPS task JMFIT (the FWHM was $9.6 \times 6.5$ mas). This revealed an extended component estimated at $2.70 \pm 0.10 \times 1.2 \pm 0.05$ mas $(\mathrm{FWHM})$, with a position angle of $140( \pm 2)^{\circ}$.

\footnotetext{
${ }^{2}$ see - http://www.geant2.net
} 


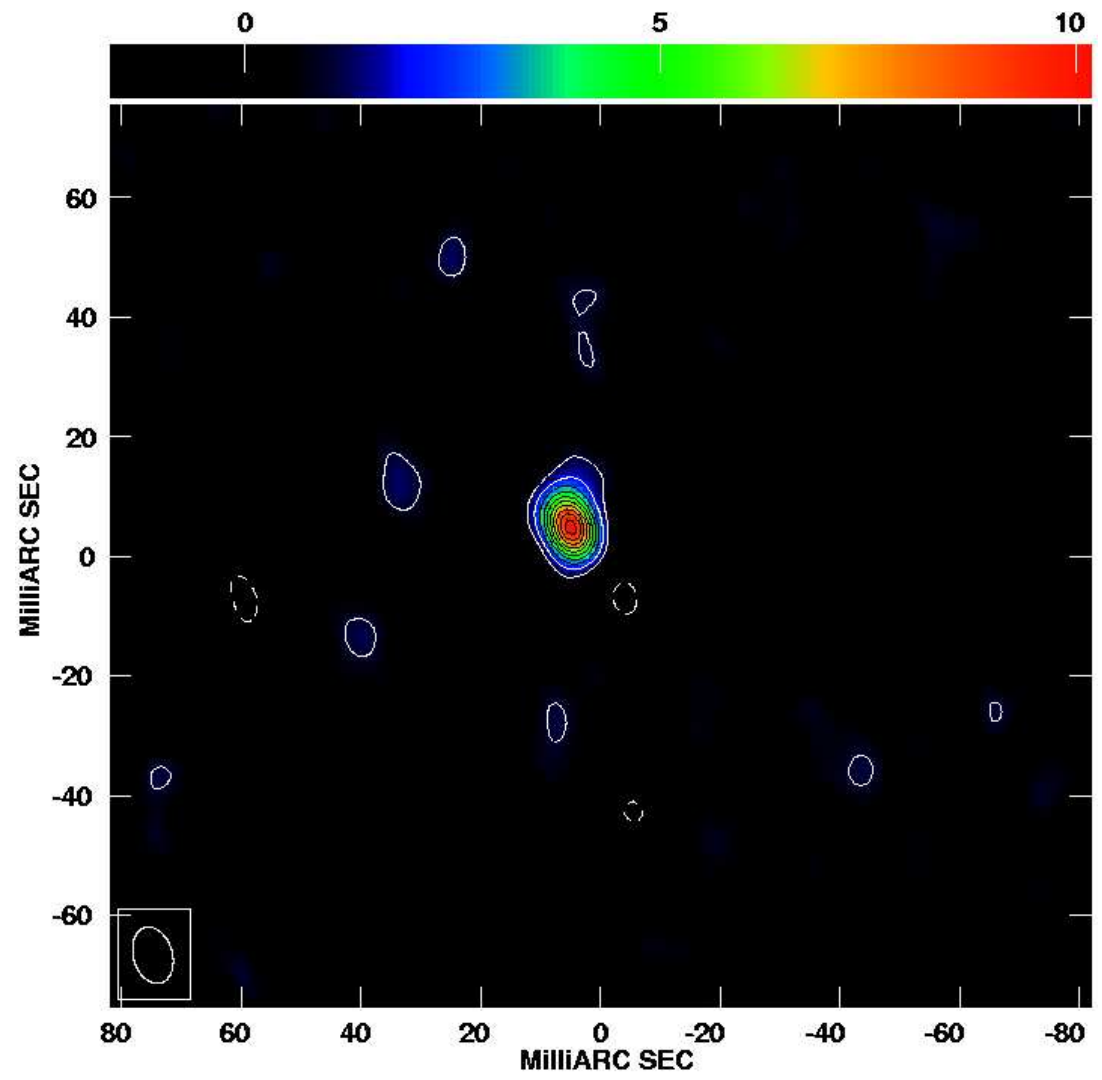

Figure 2: e-EVN map of GRS $1915+105$ at $5 \mathrm{GHz}$ using 6 telescopes centred at R.A. $19^{\mathrm{h}} 15^{\mathrm{m}}(11.548 \pm$ $0.001)^{\mathrm{s}}$ and Dec. $10^{\circ} 56^{\prime}(44.71 \pm 0.01)^{\prime \prime}(\mathrm{J} 2000)$ on 21 April 2006. Contour levels are (-1, 1, 2, 4, 6, 8, 10) times $1 \mathrm{mJy}$ per beam, with an rms of $0.3 \mathrm{mJy}$. The beam size was $9.6 \times 9.5$ mas and was deconvolved with the source revealed an extended component of $2.7 \times 1.2$ mas at a position angle of $140( \pm 2)^{\circ}$.

This is similar to the P.A. of the large scale jets previously observed [6]. The total integrated radio flux density was $11.1( \pm 0.6) \mathrm{mJy}$.

The Ryle Radio Telescope and the RXTE all sky monitor ${ }^{3}$ regularly observes GRS 1915+105. Fig. 1 shows the XRB flux density between $2006 \mathrm{Jan}-$ April at $15 \mathrm{GHz}$ and $2-10 \mathrm{keV}$ in the bottom and middle plots respectively. The top plot in Fig. 1 shows the X-ray spectral hardness radio $\left(\frac{5-12 \mathrm{keV}}{3-5 \mathrm{keV}}\right)$.

The date of the e-VLBI observation (MJD 53846), is marked. The flux entered a period of relative radio quietness in the two weeks before the e-VLBI observation. During the observation, the ASM count rate was about $40 \mathrm{~s}^{-1}$, which is $\sim 0.5 \mathrm{crab}$ [10]. The X-ray spectral hardness changed just before the epoch of the observation to a slightly softer state.

\section{Discussion amd Conclusions}

Fig. 3 shows the Ryle Radio Telescope data on 2006 April 21 between 01:27 - 08:32 UT. A fhre of $40 \mathrm{mJy}$ was detected, which quickly decayed to $\sim 20 \mathrm{mJy}$ within 4.5 hours. Assuming that

\footnotetext{
${ }^{3}$ quick-look results provided by the ASM/RXTE team
} 


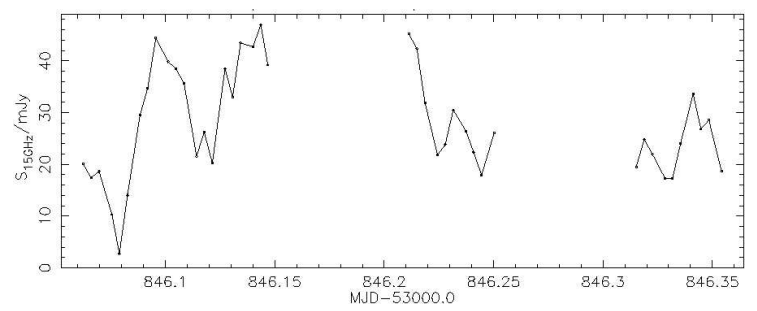

Figure 3: Ryle Radio Telescope 15 GHz flux monitoring of GRS 1915+105, observed at 2006 April 21 01:27 - 08:32 UT.

the flare expands isotropically; the minimum energy in the magnetic field and energetic electrons can be calculated assuming equipartition within a synchrotron radiation field [4]. For a distance of $11 \mathrm{kpc}$ [6] the minimum energy is $2 \times 10^{41} \mathrm{ergs}$. Using the deconvolved size from the image rather than assuming spherical expansion, we find a minimum energy of $1 \times 10^{40} \mathrm{ergs}$, a lower value due to the source being collimated.

The radio emission for this and similar weak flares (see Fig. 1) decays rapidly ( $<1$ day). This is unlike the major flares studied by the VLA and MERLIN $[6,12,11]$ where the decay is over several days and the ejecta can be followed for up to 2 months after the flare. The behaviour of the strong fhre is consistent with the shock-in-jet model [11]; however the short fares seem to show the charateristic of an expanding source without continuous ejection of relativistic electrons.

The relationship between the radio and X-ray fhre is consistent with that for other black holes in the hard state [7]. Such sources have compact jets and fht spectra. The spectrum measured in our observations between 5 and $15 \mathrm{GHz}$ is fht or slightly inverted, and futhermore the source is aligned with the P.A. of observed major ejections [12]. This further supports the idea that radio jets are present when X-ray binaries are in the low/hard state. We note that though still in the hard state, figure 1 shows that the hardness ratio falls slightly in coincidence with the occurrence of a weak radio flare.

This work clearly shows the ability of the e-EVN to produce high resolution radio maps in real time, hence eliminating the need of tape/disc recording. The use of e-VLBI enabled us to obtain images within approximately a day of the VLBI run, rather than the many weeks needed for conventional recording based observations. In the future, e-VLBI transmission rates will keep increasing with network development, yielding higher sensitivities and longer baselines will be achieved with the addition of more telescopes to the network. Announcements of opportunity with information on applications are made on the e-VLBI web site (http://www.evlbi.org/evlbi) currently every $\sim 2$ months. This is a positive step in the development of a more dynamic and fexible network.

\section{References}

[1] Briggs, D. S. 1995, Bulletin of the American Astronomical Society, 27, 1444

[2] Castro-Tirado, A. J., Brandt, S., \& Lund, N. 1992, IAUC., 5590, 2

[3] Dhawan, V., Mirabel, I. F., \& Rodríguez, L. F. 2000, ApJ, 543, 373

[4] Fender, R. P. 2006, In "Compact stellar X-ray Sources", eds. Lewin, W. H. G. and Klis, M. van der 
[5] Fender, R. P., Gallo, E., \& Jonker, P. G. 2003, MNRAS, 343, L99

[6] Fender, R. P., Garrington, S. T., McKay, D. J., Muxlow, T. W. B., Pooley, G. G., Spencer, R. E., Stirling, A. M., \& Waltman, E. B. 1999, MNRAS, 304, 865

[7] Gallo, E., Fender, R. P., \& Pooley, G. G. 2003, MNRAS, 344, 60

[8] Greiner, J., Cuby, J. G., \& McCaughrean, M. J. 2001, Nat, 414, 522

[9] Greiner, J., Cuby, J. G., McCaughrean, M. J., Castro-Tirado, A. J., \& Mennickent, R. E. 2001, A\&A, 373, L37

[10] Levine, A. M., Bradt, H., Cui, W., Jernigan, J. G., Morgan, E. H., Remillard, R., Shirey, R. E., \& Smith, D. A. 1996, ApJL, 469, L33

[11] Miller-Jones, J. C. A., McCormick, D. G., Fender, R. P., Spencer, R. E., Muxlow, T. W. B., \& Pooley, G. G. 2005, MNRAS, 363, 867

[12] Mirabel, I. F., \& Rodriguez, L. F. 1994, Nat, 371, 46 